\title{
Comparison between Two Types of Anaerobic Speed Endurance Training in Competitive Soccer Players
}

\author{
by \\ Magni Mohr1,2, Peter Krustrup ${ }^{3,4}$
}

\begin{abstract}
The purpose of the present study was to examine the effects of additional in-season speed endurance production versus speed endurance maintenance training regimes on performance in competitive male soccer players. In a randomised controlled trial 18 male sub-elite players were exposed to additional speed endurance production (SEP) or speed endurance maintenance (SEM) training (two additional sessions/wk for 4 weeks) during the competitive season. Players performed the Yo-Yo intermittent recovery level 2 test (YYIR2) and a repeated sprint test (RST) pre-and postintervention. Yo-Yo IR2 performance increased $(p<0.001)$ by $50 \pm 8 \%$ and $26 \pm 5 \%$ in SEP and SEM, respectively, with greater ( $p=0.03$ ) improvement in SEP. RST performance improved by $2.1 \pm 0.3 \%$ and $1.3 \pm 0.4 \%$ in SEP and SEM, respectively, while the RST fatigue index decreased $(4.4 \pm 0.8$ to $3.4 \pm 0.5 \% ; p<0.04)$ in SEP only. Peak and average speed during training were higher $(p<0.001)$ in SEP than in SEM $(24.5 \pm 0.3$ vs $19.2 \pm 0.3$ and $15.5 \pm 0.1$ $\mathrm{km} \cdot \mathrm{h}-1$ vs $9.4 \pm 0.1 \mathrm{~km} \cdot \mathrm{h}-1)$. Additional in-season anaerobic speed endurance production and maintenance training improves high-intensity exercise performance in competitive soccer players with superior effects of speed endurance production training.
\end{abstract}

Key words: adaptation effects, exercise testing, GPS, football.

\section{Introduction}

Soccer involves multiple high-intensity actions provoking fatigue during the most intense periods of play (Bradley et al., 2009; Mohr et al., 2003). During these game intervals, the anaerobic energy turnover and muscle recruitment are high (Krustrup et al., 2006a), which may exhaust the physiological mechanisms that counteract fatigue (McKenna et al., 2008). Thus, high intensity training regimes are of great importance for competitive soccer and similar team sport athletes.

Numerous studies have compared different types of high-intensity training protocols (Iaia and Bangsbo, 2010). For example, FerrariBravo et al. (2008) demonstrated that repeated shuttle sprint training induced superior performance effects compared to traditional aerobic high intensity interval training in trained soccer players. Mohr et al. (2007) reported that speed endurance training improved fatigue resistance during intense intermittent exercise and repeated sprint ability to a greater extent than speed training. Thus, exercise performance responses are highly dependent on the type of

1 - Faculty of Natural and Health Sciences, University of the Faroe Islands, Tórshavn, Faroe Islands..

2 - Centre for Health and Human Performance, Department of Food and Nutrition, and Sport Sciences, University of Gothenburg,

Gothenburg, Sweden.

3 - Department of Nutrition, Exercise and Sports, Section of Human Physiology, Copenhagen Centre for Team Sport and Health, University of Copenhagen, Denmark.

4 - Sport and Health Sciences, College of Life and Environmental Sciences, St. Luke's Campus, University of Exeter, Exeter, United Kingdom. 
high-intensity training performed.

There are two sub-categories of anaerobic speed endurance training (Bangsbo et al., 2006), i.e., speed endurance production training and speed endurance maintenance training. Speed endurance production training is conducted at very high intensities during approximately $30 \mathrm{~s}$ exercise intervals interspersed by 2-3 $\mathrm{min}$ rest periods to ensure sufficient recovery prior to undertaking the next exercise bout. In soccer, speed endurance production training is therefore usually organized as multiple series of specific near-maximal effort runs with the inclusion of relevant technical challenges (Ingebrigtsen et al., 2013). This type of training has been shown to induce marked increases in high-intensity performance in trained runners (Iaia et al., 2008, 2009) and soccer players (Ingebrigtsen et al., 2013; Thomassen et al., 2009). In contrast, speed endurance maintenance training usually involves small-sided games with few participants $(2 \mathrm{v} 2-4 \mathrm{v} 4)$ in 30-60 s intervals with similar recovery times to induce a gradual onset of fatigue in cumulative bouts which is suggested as an efficient approach for training fatigue resistance during intense exercise (Bangsbo et al., 2006). Exercise intensity in speed endurance maintenance training is therefore generally lower than in speed endurance production training and differences in exercise intensity and a work-to-rest ratio between the two training categories are likely to promote different adaptive changes.

Physical capacity in soccer players is generally improved during the pre-season preparation phase, but has been shown to fluctuate markedly within the competitive season (Mohr and Krustrup, 2014). Furthermore, it has been shown in elite soccer that physical capacity is enhanced during the season by the inclusion of additional sessions of high-intensity training (Bangsbo and Mohr, 2014), although it is currently unknown how the addition of two different types of anaerobic speed endurance training will affect physical capacity in competitive soccer players.

Thus, the principal aim of the present study was to compare the effects of a 4 week inseason period with additional anaerobic speed endurance production or additional speed endurance maintenance training on high intensity exercise performance in moderately trained competitive soccer players. It was hypothesized that players exposed to the two types of anaerobic training would display different performance and training responses.

\section{Material and Methods}

\section{Participants}

Eighteen male university soccer players (age $19 \pm 1$ yrs, body height $1.79 \pm 0.06 \mathrm{~m}$, body mass $79.4 \pm 4.4 \mathrm{~kg}$ ) took part in the study. All participants volunteered and consented to the testing protocol. The number of participants was selected from power calculation of the primary outcomes based on previous studies from the research group. The players normally trained twice weekly and played two competitive games a week during the season lasting from September to May. The two $90 \mathrm{~min}$ training sessions consisted of a warm-up followed by speed and agility training. The different types of small-sided games with the physical, technical and tactical focus were conducted. The players had at least eight years of continuous soccer training experience. The participants gave written consent to participate; the study conformed to the code of ethics of the Declaration of Helsinki and was approved by the ethical committee of the University of Exeter.

\section{Design}

To examine the effect of two different types of anaerobic speed endurance training, the competitive soccer players were randomly divided into two groups with either speed endurance production (SEP) or speed endurance maintenance (SEM) training added to their regular training program. The intervention lasted 4 weeks according to other comparable speed endurance training studies (Iaia et al., 2008). The activity pattern during training was monitored in order to be able to distinguish between the two groups. Pre and post intervention the players performed intermittent fitness tests to measure potential performance-enhancing effects of the two training protocols.

\section{Experimental Procedures}

All participants completed the Yo-Yo intermittent recovery level 2 test (Krustrup et al., 2006b) and a repeated sprint test as previously described (Krustrup et al., 2006a) before and after a 4-week training intervention. Prior to the intervention the players were randomly assigned by the researchers to two separate training 
groups: anaerobic speed endurance production training (SEP; $\mathrm{n}=9$ ) and anaerobic speed endurance maintenance training (SEM; $n=9)$. Each training group performed eight sessions (twice a week for 4 weeks) of either SEP or SEM in addition to regular training over a 4 week inseason period.

The Yo-Yo IR2 was performed indoors on a wooden surface and consisted of $2 \times 20 \mathrm{~m}$ shuttle runs, gradually increasing the pace through the test levels signaled by audio beeps (Bangsbo and Mohr, 2012). Prior to the test the subjects performed a standardized warm-up as described by Krustrup et al. (2006b). Between each running bout, participants had a $10 \mathrm{~s}$ active recovery period comprising $2 \times 5 \mathrm{~m}$ jogging. When the subjects failed twice to reach the finish line in time before the audio beep, the level achieved was recorded and used to calculate the distance covered.

The repeated sprint test (RST) was performed outdoors on an artificial grass surface. Each test comprised $5 \times 30 \mathrm{~m}$ sprints separated by 25 s active recovery during which the subjects jogged back to the starting line. Each sprint result was determined by wireless infra-red timing gates (Eleiko Sport, Halmstad, Sweden) as previously described (Bangsbo and Mohr, 2012). Prior to the sprint tests, a standardized warm-up was led by the coach. All participants were familiarized with both performance tests. The tests were conducted on separate days with at least $48 \mathrm{~h}$ between each test to allow sufficient recovery. Pre- and posttraining tests were performed at the same time of the day and led by the same person. Participants were advised to eat a standardized meal 2 hours prior to testing in order to be fully hydrated and to avoid consuming alcohol the day before testing and items containing caffeine on the day of testing itself.

The training-intervention sessions were monitored using GPS devices (GPSports SPI Elite, Canberra, Australia) with a time resolution of 10 $\mathrm{Hz}$ to measure the activity characteristics and the heart rate during the drills as described by Randers et al. (2010a). This approach had been proven to be reliable to provide accurate data for team sports (Edgecomb and Norton, 2006), and to be sensitive enough to measure intensity variations during games (Randers et al., 2010b) as well as training drills (Randers et al., 2010a) in soccer. The variables recorded using GPS devices were: peak speed and average running speed, total distance covered and the heart rate. Both groups trained over a 4 week period with two sessions per week alongside their two competitive games per week. The intervention was carried out in the middle of the competitive season. Each additional training session in SEP and SEM comprised 8 bouts during the first 3 weeks, which increased to 10 bouts during the last week. SEP training consisted of individual drills with balls placed in specific positions to reflect game situations (Bangsbo and Mohr, 2014), with an exercise-to-rest ratio of 1:5 (Bangsbo et al., 2006). The duration of each bout was $30 \mathrm{~s}$, with a passive rest period of $150 \mathrm{~s}$, and the players were instructed to run all-out during each bout and were verbally encouraged by the researchers. SEM training included small-sided games of $2 \mathrm{v} 2$ with goalkeepers in both goals, on a 20×20 m field with an exercise-to-rest ratio of 1:1 (Bangsbo et al., 2006). The players were instructed to work as hard as possible and were verbally encouraged by the researchers. This type of small-sided game is commonly used in soccer fitness training (Dellal et al., 2012). Duration of each bout was $45 \mathrm{~s}$, with a $45 \mathrm{~s}$ rest period. The training intervention was carried out after a regular soccer warm-up and was conducted by the researchers, who are trained soccer coaches.

\section{Statistical Analyses}

Data are presented as means \pm SE. Differences in test performance pre and post training intervention were evaluated using paired t-tests, while differences between the training interventions were evaluated using unpaired $t$ tests. Differences in activity profiles and training responses over the 4 weeks were determined using a two-way ANOVA with repeated measurements. A significance level of 0.05 was chosen.

\section{Results}

\section{Performance Response}

At baseline Yo-Yo IR2 performance was $680 \pm 68$ and $693 \pm 52 \mathrm{~m}$, respectively, in the SEP and SEM group and increased $(p<0.05)$ to $978 \pm 57$ $\mathrm{m}(49.7 \pm 8.4 \%)$ and $858 \pm 48 \mathrm{~m}(25.8 \pm 5.0 \%)$ during the training period with greater $(p<0.05)$ improvement in the SEP than SEM group (Figure 1). Pre-intervention mean sprint time in the RST 
was $4.45 \pm 0.05$ and $4.41 \pm 0.07 \mathrm{~s}$ in the SEP and SEM group, respectively, and was improved by $2.1 \pm 0.3$ and $1.3 \pm 0.4 \%$ post intervention (Figure 1). The fatigue index during the RST was reduced $(p<0.05)$ from $4.4 \pm 0.8$ to $3.4 \pm 0.5 \%$ in the SEP but unchanged in SEM group (Figure 1). Peak sprint time was $4.34 \pm 0.05$ and $4.32 \pm 0.06 \mathrm{~s}$ in the SEP and SEM group, respectively, before the training intervention and was improved by $1.7 \pm 0.3 \%$ in the SEP group after the training period while it remained unchanged in the SEM group (Figure 1).

\section{Training Response}

Peak and mean speed reached during the exercise intervals over the entire training period were $24.5 \pm 0.3$ and $15.5 \pm 0.1 \mathrm{~km} \cdot \mathrm{h}^{-1}$ in the SEP group, which was higher $(p<0.05)$ than in the SEM group $\left(19.2 \pm 0.3\right.$ and $9.4 \pm 0.1 \mathrm{~km} \cdot \mathrm{h}^{-1}$; Figures
$2 \mathrm{~A}$ and $2 \mathrm{~B}$ ). Average distance covered per exercise bout was $126 \pm 1$ and $117 \pm 1 \mathrm{~m}$ in SEP and SEM groups, with no differences between groups (Figure 3). Peak speed, average speed and average distance covered per exercise bout increased $(p<0.05)$ progressively over the 4 weeks in the SEP, however, no changes occurred in these variables over time in the SEM group (Figures 2 and 3).

No differences were observed in average heart rates during the training intervention between SEP and SEM groups (160 \pm 1 and $159 \pm 1$ btp. $\mathrm{min}^{-1}$, respectively), which corresponded to 81.2 and $79.9 \%$ HR peak, respectively. However, an average peak HR during the exercise intervals was $180 \pm 1 \mathrm{btp} \cdot \mathrm{min}^{-1}$, or $91.4 \% \mathrm{HR}_{\max }$, which was higher $(p<0.05)$ than corresponding values during SEM $\left(171 \pm 1 \mathrm{btp} \cdot \mathrm{min}^{-1}\right.$ and $85.9 \%$ HRmax $)$.

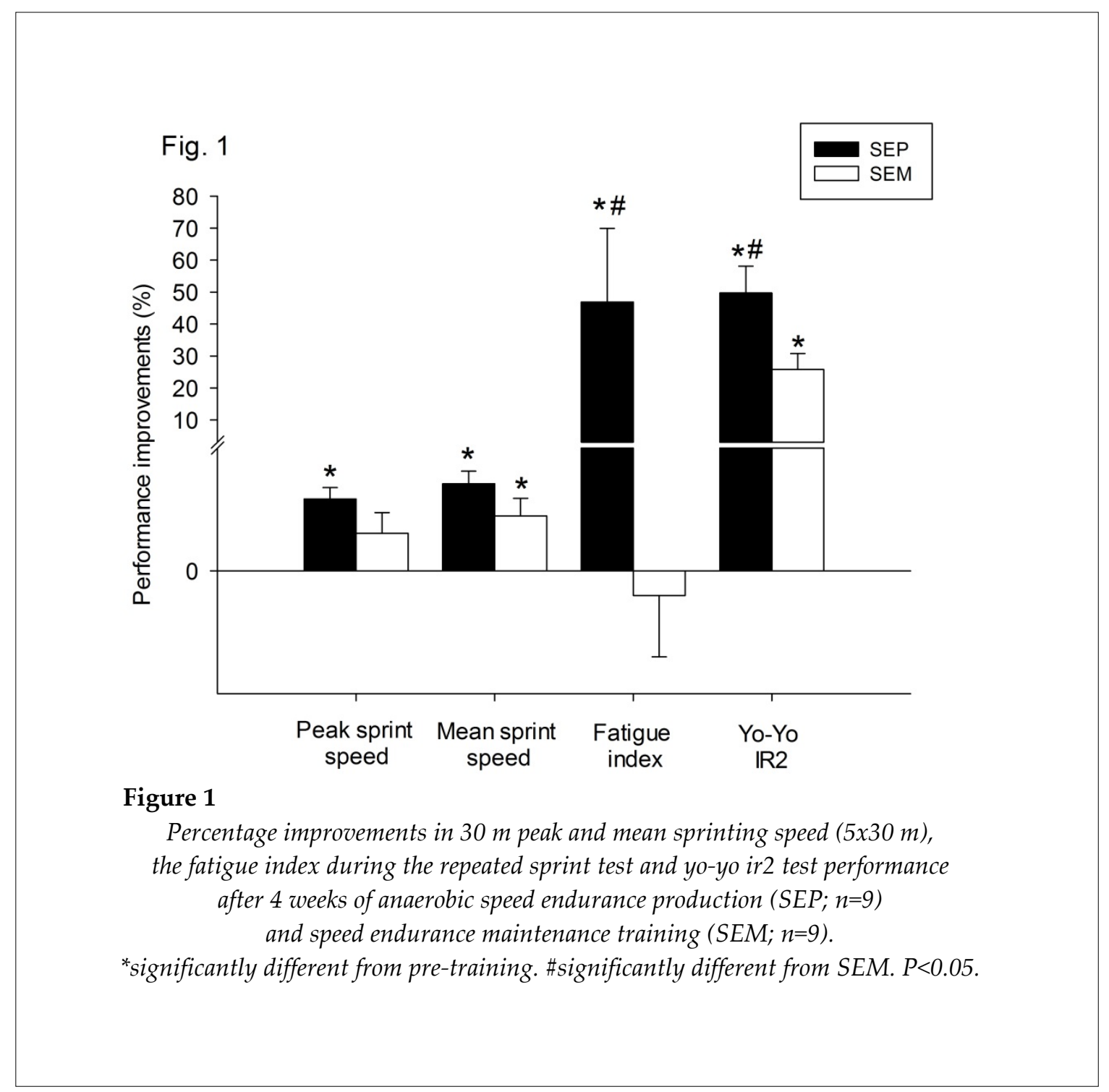


Fig. 2

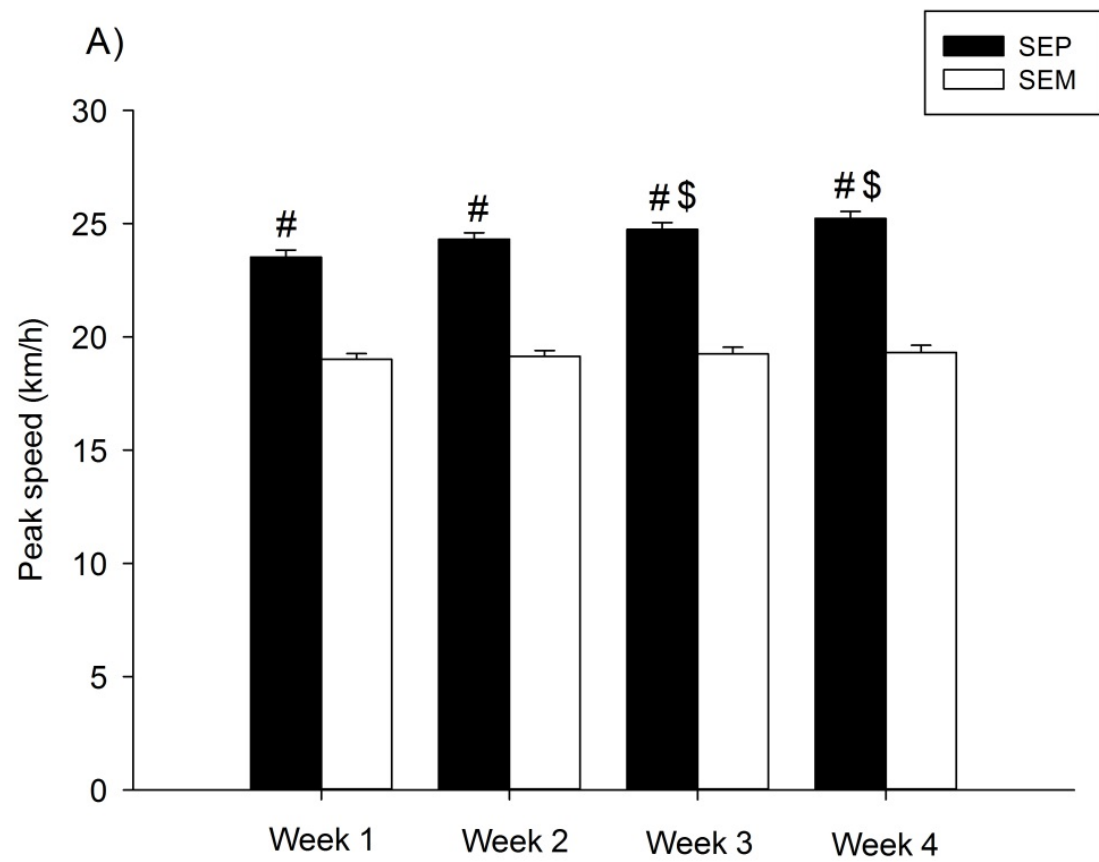

B)

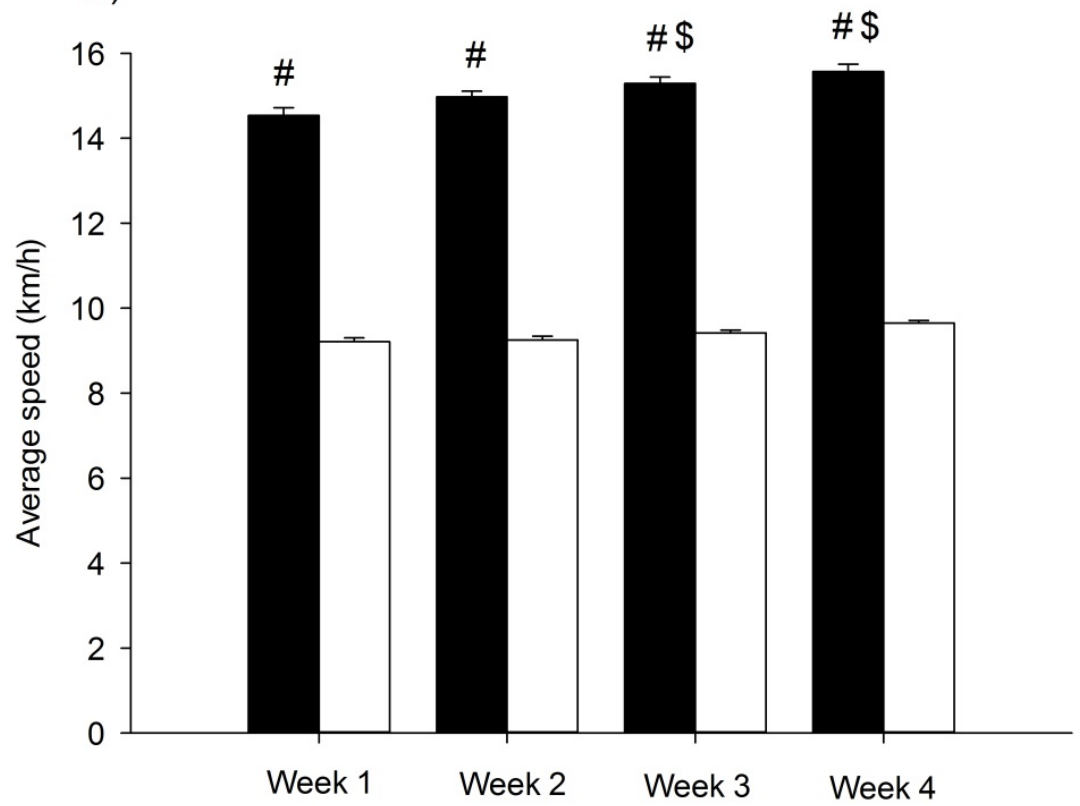

Figure 2

Peak (a) and average (b) running speed per exercise interval during

all training sessions over the 4 week training period with anaerobic speed endurance production (SEP; $n=9)$ and speed endurance maintenance training (SEM; $n=9$ ). \#significantly different from SEM. $\$$ significantly different from weeks 1 and 2. $P<0.05$ 
Fig. 3

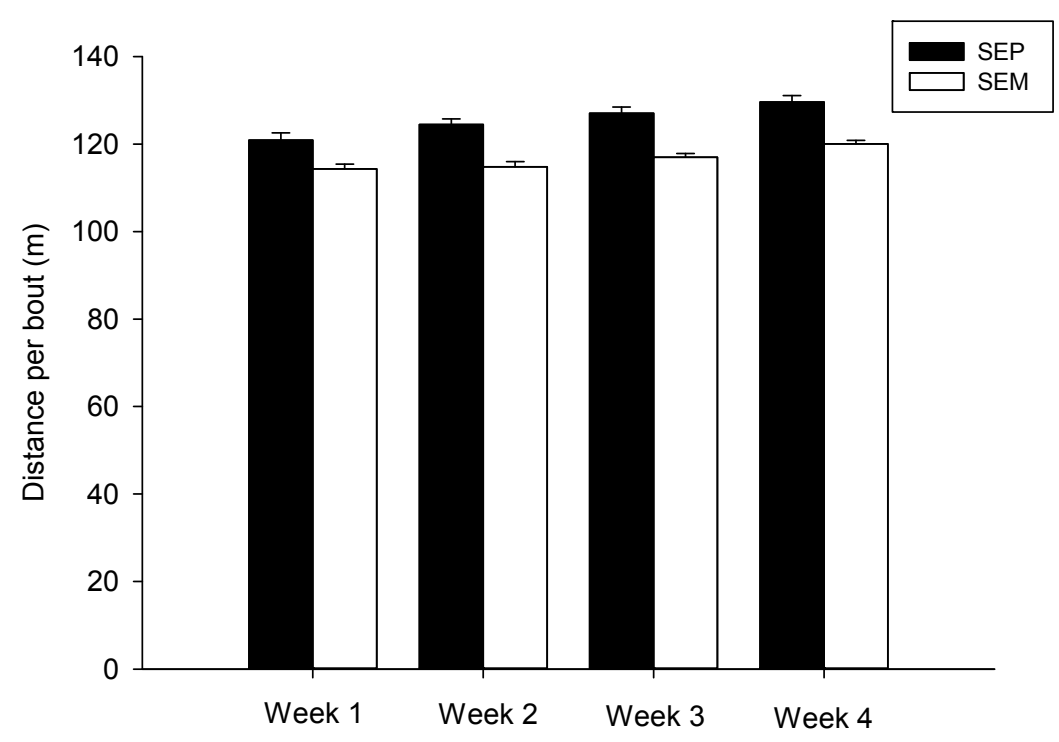

Figure 3

Average distance covered per exercise interval during all training sessions over the 4 week training period with anaerobic speed endurance production (SEP; $n=9)$ and speed endurance maintenance training (SEM; $n=9$ )

\section{Discussion}

The principal findings of the present study were that anaerobic speed endurance production training induced a greater effect on Yo-Yo IR2, repeated sprint ability and peak sprinting performance in moderately trained soccer players, compared to anaerobic speed endurance maintenance training. In addition, speed endurance production training organized as individual soccer drills produced markedly higher peak and average running speed, as well as a higher peak heart rate response, than speed endurance maintenance training conducted as $2 \mathrm{v} 2$ small-sided games, while there was no difference in distance covered between the two training regimes.

A previous study investigating welltrained youth soccer players reported that anaerobic speed endurance training effectively improved intermittent exercise capacity (Ingebrigtsen et al., 2013). However, to our knowledge the present study is the first to compare the effects of two different types of anaerobic speed endurance training. The improvement in Yo-Yo IR2 performance observed over 4 weeks of in-season training was high for speed endurance maintenance training $(26 \%)$ and even higher for speed endurance production training (50\%). Such improvements are comparable to improvements reported during a full pre-season period in well-trained elite soccer players (Krustrup et al., 2006b) and greater than previously observed in studies investigating short-term in-season anaerobic speed endurance training (11\%; Ingebrigtsen et al., 2013) or shortterm out-of-season anaerobic training (25\%; Thomassen et al., 2009). It should be emphasized that the subjects in the present study had a Yo-Yo IR2 performance that was $10-50 \%$ worse than in 
elite soccer players (Krustrup et al., 2006b; Mohr and Krustrup, 2013), but still $40 \%$ better than the high-level junior players and normally trained youth players investigated in previous studies with anaerobic training (Ingebrigtsen et al., 2013; Mohr et al., 2007). Considering this, it is impressive that only 4 weeks of additional anaerobic speed endurance training induced a $50 \%$ increase in Yo-Yo IR2 performance and raised the performance to levels comparable to high-level elite players (Bangsbo and Mohr, 2012).

Both groups showed similar changes in repeated sprint ability after the two training regimes, averaging $\sim 1.5-2 \%$ improvement postintervention. This is less than the $\sim 4 \%$ improvement showed in repeated sprint performance after 8 weeks of speed endurance training in untrained males (Mohr et al., 2007), but similar to the $\sim 2 \%$ improvement in repeated sprint performance after 6 weeks of repeated sprint training in trained soccer players (Ferrari-Bravo et al., 2008). In addition to a markedly greater improvement in Yo-Yo IR2 performance in the speed endurance production trained group compared with the speed endurance maintenance group, the former also demonstrated an $\sim 45 \%$ improvement in their fatigue index during the repeated sprint test, which is comparable to that shown in untrained males after 8 weeks of speed endurance production training (Mohr et al., 2009). In contrast, the fatigue index was not affected in the speed endurance maintenance group. Thus, speed endurance production training appears to be a very efficient training regime for promoting exercise tolerance during intense intermittent exercise in team sport athletes.

In the present study, the activity pattern and heart rate responses were recorded during all training sessions, and it was demonstrated that the speed endurance production players reached markedly higher peak and average running speeds as well as higher peak heart rates in comparison to the speed endurance maintenance players. Several studies have investigated the effects of speed endurance production training on fatigue resistance during high-intensity exercise and shown greater improvement compared with speed training (Mohr et al., 2007), endurance training (Iaia et al., 2008) and short-term tapering (Thomasson et al., 2009). Additional sessions of speed endurance training during a short-term inseason period can promote performance enhancements (Gunnarsson et al., 2012; Iaia and Bangsbo, 2010). The performance improvements in these studies were accompanied by elevated expression of muscle proteins, which were suggested to be important factors in counteracting fatigue development during intense intermittent exercise (Iaia and Bangsbo, 2010). In addition, these muscle variables were demonstrated to correlate to high intensity running distance in a soccer game (Mohr et al., 2016). Thus, a very high and constant exercise intensity during speed endurance production training and concomitant long recovery intervals may be speculated to be a stimuli for a wide range of adaptations in muscular systems that are important for improving fatigue resistance during intense intermittent exercise. In contrast, a relatively lower exercise intensity combined with short recovery intervals during speed endurance maintenance training does not seem to elicit such significant effects on performance as speed endurance production training.

The present study also showed that peak heart rates were significantly higher in the speed endurance production group, despite a similar average heart rate response during the two types of anaerobic speed endurance training. In a recent study by Christensen et al. (2011) that involved elite soccer players, it was shown that 2 weeks of intensified training encompassing anaerobic speed endurance production training markedly accelerated oxygen uptake kinetics, which is in line with others using intense intermittent training regimes (Krustrup et al., 2004; Laursen, 2010). In addition, a training study administering speed endurance production training to distance runners found greater improvement in running economy compared to traditional endurance training (Iaia et al., 2009). Future studies with more physiological measurements are required to explore whether adaptations in oxygen uptake kinetics and running economy may have contributed to the observed differences in performance enhancements between the speed endurance production and speed endurance maintenance groups.

A limitation of the present study is the lack of physiological measurements to support the performance improvements. Therefore, future 
studies should aim at examining the differences in muscular adaptations to speed endurance production and maintenance training in soccer. Moreover, the players in the present study were moderately trained based on their repeated sprint, peak sprint and Yo-Yo IR2 test scores (Bangsbo and Mohr, 2012). Thus, it is unknown if elite players will demonstrate similar adaptive responses.

In the speed endurance production training group mean peak speed, average speed, and distance covered per exercise bout increased progressively over the 4 week period, while these training variables remained unchanged in the speed endurance maintenance group, which may have had an impact on the performance response after the 4 week intervention. During individual fitness training drills the player is not affected by other players and it is relatively easy to ensure that the exercise intensity is kept high during the entire drill in comparison to conventional smallsided games such as 2v2 (Bangsbo and Mohr, 2014). Large individual variations have been observed in match analyses of elite players in several scientific studies (Bradley et al., 2009; DiSalvo et al., 2009; Mohr et al., 2003; Rampinini et al., 2009). Recently, similar variations were demonstrated in small-sided games (Dellal et al., 2012). Thus, traditional small-sided games may not provide the optimal protocol for all soccer players if the principles of speed endurance production training are to be upheld. Individual soccer-specific running drills with technical challenges simulating game situations may be an efficient alternative (Bangsbo and Mohr, 2014).

In the present study, $30 \mathrm{~m}$ peak sprint performance was only increased in the speed endurance training group. Others had found that the fatigue index in repeated sprint tests was inversely associated with sprint performance in the first sprint (Bishop and Edge, 2006). However, in the present study both fatigue index and peak sprint performance were improved only in the speed endurance training group and all nine participants in this group had their peak sprint performance in the first of the five sprints. Part of the increase may relate to the relatively low initial fitness level of the players, but this may also highlight the importance of intensified training in soccer.

In conclusion, this is the first study to compare the performance effects and training response between anaerobic speed endurance production and maintenance training in team sport athletes. It was demonstrated that speed endurance production training improved intense intermittent exercise capacity and repeated sprint ability to a greater extent than speed endurance maintenance training. These differences are likely to be caused by higher exercise intensity during the speed endurance production training intervention compared to speed endurance maintenance training, however, further studies are required in order to investigate the physiological mechanisms behind the observed performance adaptations.

\section{Acknowledgements}

The authors would like to thank the players, coaches and club for their committed participation. We would also like to thank Henrik Holm Andersen for grammatical advice. No financial support was granted for the study.

\section{References}

Bangsbo J, Mohr M, Krustrup P. Physical and metabolic demands of training and match-play in the elite football player. J Sports Sci, 2006; 24: 665-674

Bangsbo J, Mohr M. Fitness Testing in Football. Copenhagen: Bangsbosport, 1-167; 2012

Bangsbo J, Mohr M. Individual Training in Football. Copenhagen: Bangsbosport, 1-144; 2014

Bishop D, Edge J. Determinants of repeated-sprint ability in females matched for single-sprint performance. Eur J Appl Physiol, 2006; 97: 373-379

Bradley PS, Sheldon W, Wooster B, Olsen P, Boanas P, Krustrup P. High-intensity running in English FA Premier League soccer matches. J Sports Sci, 2009; 27: 159-168 
Christensen PM, Krustrup P, Gunnarsson TP, Kiilerich K, Nybo L, Bangsbo J. VO2 kinetics and performance in soccer players after intense training and inactivity. Med Sci Sports Exerc, 2011; 43: 1716-1724

Dellal A, Drust B, Lago-Penas C. Variation of activity demands in small-sided soccer games. Int J Sports Med, 2012; 33: 370-375

Di Salvo V, Gregson W, Atkinson G, Tordoff P, Drust B. Analysis of high intensity activity in Premier League soccer. Int J Sports Med, 2009; 30: 205-212

Edgecomb SJ, Norton KI. Comparison of global positioning and computer-based tracking systems for measuring player movement distance during Australian football. J Sci Med Sports, 2006; 9: 25-32

Ferrari-Bravo D, Impellizzeri FM, Rampinini E, Castagna C, Bishop D, Wisloff U. Sprint vs. interval training in football. Int J Sports Med, 2008; 29: 668-674

Gunnarsson TP, Christensen PM, Holse K, Christiansen D, Bangsbo J. Effect of additional speed endurance training on performance and muscle adaptations. Med Sci Sports Exerc, 2012; 44: 1942-1948

Iaia FM, Thomassen M, Kolding H, Gunnarsson TP, Wendell J, Rostgaard T, Nordsborg N, Krustrup P, Nybo L, Hellsten Y, Bangsbo J. Reduced volume but increased training intensity elevates muscle $\mathrm{Na}+-$ $\mathrm{K}+$ pump alpha1-subunit and NHE1 expression as well as short-term work capacity in humans. Am J Physiol Integr Comp Physiol, 2008; 294: R966-R974

Iaia FM, Hellsten Y, Nielsen JJ, Fernström M, Sahlin K, Bangsbo J. Four weeks of speed endurance training reduces energy expenditure during exercise and maintains muscle oxidative capacity despite a reduction in training volume. J Appl Physiol, 2009; 106: 73-80

Iaia FM, Bangsbo J. Speed endurance training is a powerful stimulus for physiological adaptations and performance improvements of athletes. Scand J Med Sci Sports, 2010; 20: 11-23

Ingebrigtsen J, Shalfawi SA, Tønnessen E, Krustrup P, Holtermann A. Performance effects of 6 weeks of aerobic production training in junior elite soccer players. J Strength Cond Res, 2013; 27: 1861-1867

Krustrup P, Hellsten Y, Bangsbo J. Intense interval training enhances human skeletal muscle oxygen uptake in the initial phase of dynamic exercise at high but not at low intensities. J Physiol, 2004; 559: 335-345

Krustrup P, Mohr M, Steensberg A, Bencke J, Kjaer M, Bangsbo J. Muscle and blood metabolites during a soccer game: implications for sprint performance. Med Sci Sports Exerc, 2006a; 38: 1165-1174

Krustrup P, Mohr M, Nybo L, Jensen JM, Nielsen JJ, Bangsbo J. The Yo-Yo IR2 test: physiological response, reliability, and application to elite soccer. Med Sci Sports Exerc, 2006b; 38: 1666-1673

Laursen PB. Training for intense exercise performance: high-intensity or high-volume training? Scand J Med Sci Sports, 2010; Suppl 2: 1-10

McKenna MJ, Bangsbo J, Renaud JM. Muscle $\mathrm{K}+, \mathrm{Na}+$ and $\mathrm{Cl}$ disturbances and $\mathrm{Na}+-\mathrm{K}+$ pump inactivation: implications for fatigue. J Appl Physiol, 2008; 104: 288-95

Mohr M, Krustrup P, Bangsbo J. Match performance of high-standard soccer players with special reference to development of fatigue. J Sports Sci, 2003; 21: 519-528

Mohr M, Krustrup P, Bangsbo J. Fatigue in soccer: A brief review. J Sports Sci, 2005; 23: 593-599

Mohr M, Krustrup P, Nielsen JJ, Nybo L, Rasmussen MK, Juel C, Bangsbo J. Effect of two different intense training regimes on skeletal muscle ion transport systems and fatigue development. Am J Physiol Reg Integr Comp Physiol, 2007; 292: R1594-R1602

Mohr M, Krustrup P. Yo-Yo Intermittent Recovery test performances within an entire football league during a full season. J Sports Sci, 2013; 32: 315-327

Mohr M, Thomassen M, Girard O, Racinais S, Nybo L. Muscle variables of importance for physiological performance in competitive football. Eur J Appl Physiol, 2016; 116: 251-262

Randers MB, Nybo L, Petersen J, Nielsen JJ, Christensen L, Bendiksen M, Brito J, Bangsbo J, Krustrup P. Activity profile and physiological response to football for untrained males and females, elderly and 
youngster: influence of the number of players. Scand J Med Sci Sports, 2010a; 20: 14-23

Randers MB, Mujika I, Hewitt A, Santisteban J, Bischoff R, Solano R, Petrole E, Krustrup P, Mohr M. Application of four different football match analyzing systems - a comparative study. J Sports Sci, 2010b; 28: 171-182

Rampinini E, Impellizzeri FM, Castagna C, Coutts AJ, Wisløff U. Technical performance during soccer matches of the Italian Serie A league: effect of fatigue and competitive level. J Sci Med Sport, 2009; 12: 227-233

Thomassen M, Christensen PM, Gunnarsson TP, Nybo L, Bangsbo J. Effect of 2-wk intensified training and inactivity on muscle $\mathrm{Na}+-\mathrm{K}+$ pump expression, phospholemman (FXYD1) phosphorylation, and performance in soccer players. J Appl Physiol, 2009; 108: 898-905

\section{Corresponding author:}

\section{Magni Mohr}

Faculty of Natural and Health Sciences

University of the Faroe Islands

Jónas Broncks gøta 25. 3rd floor

Tórshavn, Faroe Islands

Tel.: + 298292270

E-mail: magnim@setur.fo 